\title{
Efficient high-fidelity quantum computation using matter qubits and linear optics
}

\author{
Sean D. Barrett* and Pieter Koli \\ Hewlett Packard Laboratories, Filton Road, Stoke Gifford, Bristol BS34 8QZ, UK
}

(Dated: February 1, 2008)

\begin{abstract}
We propose a practical, scalable, and efficient scheme for quantum computation using spatially separated matter qubits and single photon interference effects. The qubit systems can be NV-centers in diamond, Pauli-blockade quantum dots with an excess electron or trapped ions with optical transitions, which are each placed in a cavity and subsequently entangled using a double-heralded single-photon detection scheme. The fidelity of the resulting entanglement is extremely robust against the most important errors such as detector loss, spontaneous emission, and mismatch of cavity parameters. We demonstrate how this entangling operation can be used to efficiently generate cluster states of many qubits, which, together with single qubit operations and readout, can be used to implement universal quantum computation. Existing experimental parameters indicate that high fidelity clusters can be generated with a moderate constant overhead.
\end{abstract}

PACS numbers: 32.80.-t, 78.70.-g

Quantum computation (QC) offers a potentially exponential computational speed-up over classical computers, and many physical implementations have been proposed. Particularly promising proposals are those in which unitary operations and readout in matter qubits are implemented via laser-driven optical transitions. Examples are the original ion-trap proposal [1], NV-centers in diamond 2], and schemes utilizing the Pauli-blockade effect in quantum dots with a single excess electron 3, 4]. Single qubit operations and readout, using a combination of optical and RF control fields, have already been demonstrated in ion trap and NV-diamond systems [2, 5, 6], while a number of promising techniques for optically addressing quantum dot spin qubits have been proposed [3, 4]. In all these cases, the ratio of the single qubit operation time to the intrinsic decoherence times suggests that very high fidelity operations are possible.

However, there are substantial difficulties in scaling these implementations to the large numbers of qubits required for useful QC. Multi-qubit gates are facilitated by a direct interaction between qubits. Thus adding a new qubit to a quantum register, together with the associated control fields, necessarily modifies the Hamiltonian of the system. This can mean that, as more qubits are added, logic gate implementations become progressively more complex, and furthermore, new decoherence channels can be introduced. Furthermore, the need to optically address individual qubits (e.g. in NV diamond or quantum dot systems) can lead to seemingly contradictory system requirements: the qubits need to be sufficiently well separated to be resolved by the optical field, but must be close enough such that two-qubit logic can be implemented via the inter-qubit interaction.

A potentially promising solution to these scaling challenges is to perform distributed qunatum computing, in which the matter qubits are spatially separated. In this case, there is no direct interaction between the qubits. Instead, Entangling Operations (EOs) between qubits are

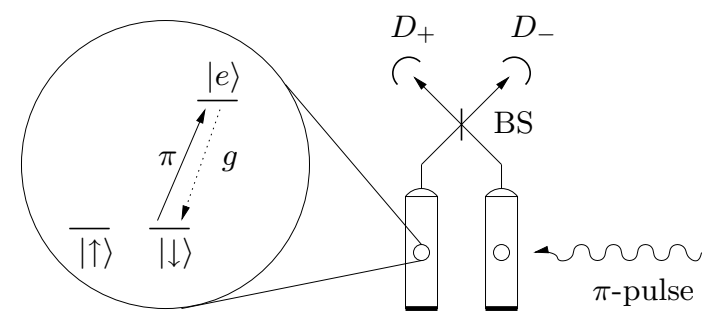

FIG. 1: In the circle: The qubit system $\{|\uparrow\rangle,|\downarrow\rangle\}$ with the excited state $|e\rangle$. The $\pi$-pulse affects only the transition $|\downarrow\rangle \rightarrow$ $|e\rangle$, and the emission of a photon into the cavity mode brings the excited state back to the qubit state $|\downarrow\rangle$. Using a pair of leaky cavities with this system and conditioning on a singlephoton in the detectors $D_{+}$or $D_{-}$creates entangled qubit states. The 50:50 beam splitter (BS) is used to erase the which-path information.

implemented via single photon interference effects. A number of schemes to entangle pairs of distant qubits in this way have been proposed $7,8,19,10,11,12$. Recently, it has been shown that unitary logic gates can be can be performed in this manner [13, 14]. However, the latter schemes are either inherently non-deterministic 14] or are sensitive to photon loss or photodetector inefficiency [13] and it is not clear whether they can be used for scalable QC. Other schemes using single photon interference effects together with local two-qubit unitary operations have also been proposed [15, 16].

In this Letter, we propose a fully scalable scheme for distributed QC using individual matter qubits assuming only single qubit operations. Our scheme is robust to photon loss and other sources of errors, and uses optical transitions of the qubit system, together with linear optics and photodetection to entangle pairs of spatially seperated matter qubits in a non-deterministic manner. A key observation is that even such a non-deterministic EO is sufficient for scalable QC: our EO can be used to efficiently generate cluster states of many qubits, which, 
together with single qubit operations and measurements, are capable of universal QC 17]. In the context of linear optics QC [18], it has recently been shown 19, 20, 21. that the cluster state model can be used to significantly reduce the resource overheads required for scalable QC.

We consider matter systems comprised of two longlived, low lying states $|\uparrow\rangle$ and $|\downarrow\rangle$, and one excited state $|e\rangle$, in an $L$-configuration (see Fig. 1). The system is constructed in such a way that an optical $\pi$-pulse will induce the transformation $|\downarrow\rangle \rightarrow|e\rangle$ and $|\uparrow\rangle \rightarrow|\uparrow\rangle$. The transition $|\uparrow\rangle \leftrightarrow|e\rangle$ is forbidden, e.g., by a selection rule. The states $|\uparrow\rangle$ and $|\downarrow\rangle$ represent the logical qubit states $|0\rangle$ and $|1\rangle$ respectively. We assume that high fidelity single qubit operations and measurements can be performed on these logical qubits. Physical systems that have a suitable level structure include NV-centers in diamond 2], quantum dots with a single excess electron 3, 4], and various trapped ion and atomic systems. Each such system is embedded in a separate optical cavity, such that only the $|\downarrow\rangle \leftrightarrow|e\rangle$ transition is coupled to the cavity mode. One end of each cavity is leaky, with the leakage rate of the $i^{\text {th }}$ cavity given by $2 \kappa_{i}$. The light escaping from the cavities is mixed on a 50:50 beam splitter, the output modes of which are monitored by two vacuumdiscriminating detectors, $D_{+}$and $D_{-}$, with efficiency $\eta$.

The scheme proceeds as follows. Firstly, both qubits are prepared in the state $|+\rangle=(|\uparrow\rangle+|\downarrow\rangle) / \sqrt{2}$ using local unitaries. We then implement the following sequence of operations: i) Apply an optical $\pi$-pulse to each qubit, coherently pumping the population in the $|\downarrow\rangle$ state into the $|e\rangle$ state; $i i)$ Wait for up to a time $t_{\text {wait }}$ for a photodetection event in either $D_{+}$or $D_{-}$; iii) Wait for a further time $t_{\text {relax }}$ for any remaining excitation in the qubitcavity systems to relax; $i v$ ) Apply an $X$ operation to both qubits, coherently flipping the spins as $|\uparrow\rangle \rightarrow|\downarrow\rangle$ and $|\downarrow\rangle \rightarrow|\uparrow\rangle ; v)$ Repeat steps $i-i i i$.

Appropriate values for $t_{\text {wait }}$ and $t_{\text {relax }}$ are determined by the system parameters discussed below. If zero or two photo-detection events are observed on either round of the procedure, the scheme failed, and the qubits must be newly prepared before re-attempting the entangling procedure. On the other hand, if one (and only one) photo-detection event is observed on each round of the protocol, the scheme has succeeded, and a maximally entangled state is prepared with unit fidelity (given ideal systems). We call this technique double heralding, and it turns out to be exceedingly robust against most common experimental errors.

We analyzed the scheme in detail using the quantum trajectories formalism [22]. For clarity, we first consider the ideal case, in which the detectors have unit efficiency $(\eta=1)$, and spontaneous emission of photons from the transition $|e\rangle \rightarrow|\downarrow\rangle$ into modes other than the cavity mode is neglected. During time periods where no detector clicks are observed, the conditional state of the system, in the interaction picture, evolves smoothly ac- cording to the effective Hamiltonian $(\hbar=1)$

$$
H_{\mathrm{eff}}=\sum_{i=A, B} \frac{g_{i}}{2}\left(|\downarrow\rangle_{i i}\langle e| \hat{c}_{i}^{\dagger}+\text { H. c. }\right)-i \sum_{i=A, B} \kappa_{i} \hat{c}_{i}^{\dagger} \hat{c}_{i} .
$$

Here, $g_{i}$ denotes the Jaynes-Cummings coupling between the $|e\rangle_{i} \leftrightarrow|\downarrow\rangle_{i}$ transition and the mode of the $i^{\text {th }}$ cavity, and $\hat{c}_{i}$ is the corresponding annihilation operator. For the purpose of illustrating the ideal case, we assume that systems $A$ and $B$ are identical, such that $g_{A}=g_{B}=g$ and $\kappa_{A}=\kappa_{B}=\kappa$, and that $\kappa \geq g$.

When a single click is observed in detector $D_{ \pm}$, the state of the whole system discontinuously evolves as $|\psi(t)\rangle \rightarrow \hat{c}_{ \pm}|\psi(t)\rangle$, where $\hat{c}_{ \pm}=\left(\hat{c}_{A} \pm \hat{c}_{B}\right) / \sqrt{2}$ denotes the corresponding jump operators. Thus, after steps $i$ $i i$ of the entangling protocol, conditioned on observing a detector click at time $t_{1} \leq t_{\mathrm{wait}}$, the unnormalized state of the whole system is

$$
\begin{aligned}
\left|\tilde{\psi}\left(t_{1}\right)\right\rangle= & \alpha\left(t_{1}\right)\left|\Psi_{ \pm}\right\rangle+\alpha\left(t_{1}\right) \beta\left(t_{1}\right) \frac{|\downarrow, 0 ; e, 0\rangle \pm|e, 0 ; \downarrow, 0\rangle}{\sqrt{2}} \\
& +2 \alpha^{2}\left(t_{1}\right) \frac{|\downarrow, 0 ; \downarrow, 1\rangle \pm|\downarrow, 1 ; \downarrow, 0\rangle}{\sqrt{2}} .
\end{aligned}
$$

Here, $\left|q_{A}, p_{A} ; q_{B}, p_{B}\right\rangle$ is the state of the whole system, with $q_{A(B)}$ and $p_{A(B)}$ denoting the states of matter system $A(B)$ and cavity mode $A(B)$ respectively, $\left|\Psi^{ \pm}\right\rangle=$ $(|\downarrow, 0 ; \uparrow, 0\rangle \pm|\uparrow, 0 ; \downarrow, 0\rangle) / \sqrt{2}$ are maximally entangled states, $\alpha(t)=-i g /\left(4 \sqrt{\kappa^{2}-g^{2}}\right)\left(e^{-\Gamma_{\text {slow }} t / 2}-e^{-\Gamma_{\text {fast }} t / 2}\right)$ and $\beta(t)=\frac{1}{2}\left(1+\kappa / \sqrt{\kappa^{2}-g^{2}}\right) e^{-\Gamma_{\text {slow }} t / 2}+\frac{1}{2}(1-$ $\left.\kappa / \sqrt{\kappa^{2}-g^{2}}\right) e^{-\Gamma_{\text {fast }} / 2}$, where $\Gamma_{\text {fast }}=\kappa+\sqrt{\kappa^{2}-g^{2}}$ and $\Gamma_{\text {slow }}=\kappa-\sqrt{\kappa^{2}-g^{2}}$. In order to obtain a detector click with significant probability, $t_{\text {wait }}$ should be chosen to be a few times $\Gamma_{\text {slow }}^{-1}$.

Equation (2) implies that it may be possible to observe a second detector click on the first round of the protocol. However, realistic photo-detectors typically cannot resolve two photons arriving in quick succession [23]. Within the quantum trajectories description, this can be simulated by assuming that no information is available from either detector after the first click. After a time $t_{\text {relax }} \gg \Gamma_{\text {slow }}^{-1}$, the system decoheres to the state

$$
\rho=\frac{1}{N}\left|\Psi_{ \pm}\right\rangle\left\langle\Psi_{ \pm}\left|+\left(1-\frac{1}{N}\right)\right| \downarrow \downarrow\right\rangle\langle\downarrow \downarrow|,
$$

where $N=1+\left|\beta\left(t_{1}\right)\right|^{2}+\left|\alpha\left(t_{1}\right)\right|^{2}$. The undesirable second term in Eq. (3) is removed by applying steps $i v$ and $v$ of the entangling procedure. If a photo-detection occurs on the second round, the final state of the system is a pure, maximally entangled state. If the two clicks are observed in the same (different) detector(s), the final state is $\left|\Psi^{+}\right\rangle$ $\left(\left|\Psi^{-}\right\rangle\right)$. Each of the four possible successful outcomes occurs with probability $1 / 8$, leading to a total success probability of $p=\frac{1}{2}$.

We also analyzed the scheme in the non-ideal case, allowing for less than perfect detector efficiency $\eta<1$, and 
finite spontaneous emission into free space $\left(\gamma_{1}=\gamma_{2}=\right.$ $\gamma>0)$. These imperfections do not reduce the fidelity of the final state, but do reduce the success probability (see Fig. 2a). Note $p$ has a quadratic dependence on $\eta$, while $p$ decreases rapidly for $\gamma \gtrsim \Gamma_{\text {slow }}$.

The dominating experimental imperfections that do reduce the fidelity can be classified into three groups: (1) decoherence of the matter qubits; (2) dark counts in the detectors; and (3) imperfect mode matching of the photons incident on the beam splitter. Firstly, the effect of spin decoherence depends on the way the cluster states are generated, and can be estimated by comparing the spin decoherence time $t_{\mathrm{d}}$ with the "clock time" $t_{\mathrm{c}} \sim 10 \Gamma_{\text {slow }}^{-1}$ at which the EO can be repeated. If the preparation of cluster states is performed in parallel, the typical time overhead is $m$ clock cycles (see below). Thus the average age of a qubit the moment it is added to the cluster is $(m / 2) t_{\mathrm{c}}$, and $m \lesssim 8$ for reasonable detector efficiencies. Assuming a reasonably strong cavity qubit coupling, $g=100 \gamma$, and critically damped cavities $(g \approx \kappa)$, the size of errors due to spin decoherence is given by $\varepsilon \sim(m / 2) t_{\mathrm{c}} / t_{\mathrm{d}} \sim 0.4 \gamma^{-1} / t_{\mathrm{d}}$. For instance, for the NVdiamond system $\left(\gamma^{-1}=25 \mathrm{~ns}[24]\right.$ and $\left.t_{\mathrm{d}}=32 \mu \mathrm{s}[25]\right)$, we have $\varepsilon \sim 3 \times 10^{-4}$.

Secondly, detector dark counts on either round of the EO can lead to a spurious 'success' of the EO, which can reduce the fidelity of the entanglement. For existing APD detectors, dark count rates are typically $\Gamma_{\mathrm{dc}}<500 \mathrm{~s}^{-1}$ [26]. Dark counts can be made negligible by observing the detector output only for the window $t_{\text {wait }} \sim 3 \Gamma_{\text {slow }}^{-1}(\sim 1$ ns for NV-diamond). The probability of a spurious count is therefore $p_{\mathrm{dc}}=\Gamma_{\mathrm{dc}} t_{\text {wait }} \sim 10^{-7}$. Thus dark counts should have a negligible effect on the cluster fidelity.

Finally, imperfect mode matching of the photons emitted by the matter qubit-cavity systems reduces the fidelity, because the photons carry information regarding their origin. Non identical central frequencies, different polarizations, and spatio-temporal mode shapes of the photons can all reduce the fidelity. The frequency of the photons emitted from cavity $i$ depends on the frequencies of both the $|\downarrow\rangle_{i} \leftrightarrow|e\rangle_{i}$ transition $\left(\omega_{\downarrow e, i}\right)$ and the cavity mode $\left(\omega_{\mathrm{cav}, i}\right)$. The $\omega_{\downarrow e, i}$ 's can be tuned independently, e.g. by using local electric and magnetic fields to induce Stark and Zeeman shifts. The $\omega_{\text {cav, } i}$ 's can also be accurately and independently tuned, e.g. by using strain-tunable silica microcavities 27], or piezoelectrically tuned fibre optic microcavities [28]. The polarization of the emitted photons can be accurately matched using linear optical elements [29]. The spatio-temporal mode shapes of the emitted photons depend on the $g_{i}$ 's and $\kappa_{i}$ 's of the respective cavities. These parameters depend on the structure of the cavities, and hence are more difficult to calibrate once the cavities have been fabricated. However, we calculated that the EO is rather robust to mismatches in the $g_{i}$ 's and $\kappa_{i}$ 's (see Fig [2]): mismatches of a few percent lead to a reduction in fidelity
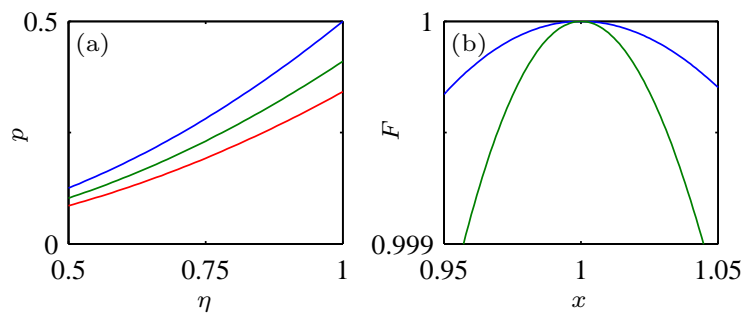

FIG. 2: (a) Probability of success $p$ versus the detector efficiency $\eta$ after both rounds of the entangling procedure. Each curve is plotted for a different value of the spontaneous emission rate $\gamma=\left\{0,0.1 \Gamma_{\text {slow }}, 0.2 \Gamma_{\text {slow }}\right\}$ (top to bottom). The other parameters used were $g=0.3$ and $\kappa=1$. (b) Fidelity of the entangled qubit pair for imperfectly mode-matched photons, calculated using the quantum trajectories formalism. Upper curve: fidelity for non identical leakage rates, $x=\kappa_{1} / \kappa_{2}$, taking $g_{1}=g_{2}$. Lower curve:fidelity for non identical coupling parameters, $x=g_{1} / g_{2}$, taking $g_{2}=0.3$ and $\kappa_{1}=\kappa_{2}=1$.

of less than $10^{-3}$. Deterministic sources of indistinguishable photons, which have similar requirements to those needed for our scheme, are currently being developed by a number of groups [24, 30, 31, 32, 33].

The next step towards scalable quantum computers is linking qubits together into cluster states, using the EO described above. A cluster state of qubits $\left\{q_{1}, q_{2}, . . q_{N}\right\}$ can be represented graphically by a collection of qubit nodes connected by edges connecting neighboring qubits, as depicted in Fig. 3a. A linear cluster of $N$ qubits (a chain) may be represented in the form $|C\rangle_{1 \ldots N}=\left(|\uparrow\rangle_{1}+\right.$ $\left.|\downarrow\rangle_{1} Z_{2}\right)\left(|\uparrow\rangle_{2}+|\downarrow\rangle_{2} Z_{3}\right) \ldots\left(|\uparrow\rangle_{N}+|\downarrow\rangle_{N}\right)$, where $Z_{i}$ represents the Pauli phase-flip operation acting on qubit $i$. Such linear clusters can be grown using our EO, as we now describe. Given a cluster $|C\rangle_{2 \ldots N}$, qubit 1 can be added to the end of the cluster by first preparing qubit 1 in the state $|+\rangle_{1} \equiv|\downarrow\rangle_{1}+|\uparrow\rangle_{1}$ and then applying the EO to qubits 1 and 2. If the EO is successful, the resulting state is of the form $\left(|\uparrow\rangle_{1}|\downarrow\rangle_{2} \pm|\downarrow\rangle_{1}|\uparrow\rangle_{2} Z_{3}\right)|C\rangle_{3 \ldots N}$, depending on whether both clicks were observed in the same detector. This can be transformed into a cluster state by applying the local operations $H_{1} X_{2}$ or $X_{1} H_{1} X_{2}$, conditional on the outcome of the EO (here $H_{i}$ is the Hadamard operation, and $X_{i}$ the Pauli operator implementing a bit flip). If the EO fails, the state of qubit 2 is, in general, unknown. However, measuring qubit 2 in the computational basis removes qubit 2 from the cluster, but projects qubits $\{3 \ldots N\}$ back into a pure cluster state. Therefore, failure of the EO causes the original cluster to shrink by 1 qubit.

Repeatedly applying the procedure described above allows long chains to be grown. However, the theoretical upper limit on the success probability of our protocol is $p=\frac{1}{2}$, and when the protocol fails, the chain shrinks by 1 qubit. Therefore, with only the procedure described above one can not create large clusters efficiently. If recy- 
a)

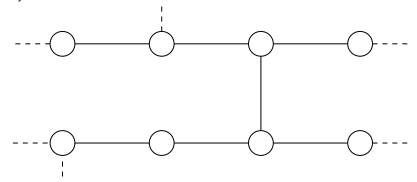

b)

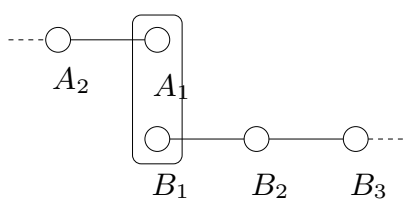

such 5-chains to a longer cluster leads to a total cost of $C_{5}=\left(2 p^{-3}+2 p^{-2}+p^{-1}+1\right) /(5 p-1)$ EOs per qubit. To minimize these costs, the collection and detection efficiencies should be maximized. For example, for $p \approx 0.24$ (or $\eta=70 \%$ with $\gamma=0$ ), we require $m=5$, and we find $C_{5}=775$. A modest improvement in detector efficiency dramatically reduces the overhead cost: for $\eta=85 \%$ and $\gamma=0$, we find $C_{4}=73.4$. Note that there may be more efficient schemes for growing linear clusters using our EO (e.g. employing recycling of small clusters [21]) which yield lower overhead costs.

In order to build linear chains into two-dimensional cluster states capable of simulating arbitrary logic networks, cross links between linear chains must be constructed 20]. Such a link can be created by first using the EO to create an $I$ shaped cluster (see Fig[3](a)) offline, for some fixed cost. Provided the arms of this $I$-cluster are sufficiently long, the EO can be used to join the $I$-cluster to a pair of linear clusters with a high probability, and therefore create a cross link between the clusters. This leads to a constant overhead cost per cross link added to the cluster. Note that other methods for creating twodimensional clusters, e.g. using microclusters [20] or redundant encoding [21], have also been proposed.

Our proposal has a number of very desirable features with respect to practical implementations. Firstly, our scheme requires only a simple level structure and singlequbit operations. Secondly, photon loss does not reduce the fidelity of the entangled state of the qubits, but merely adds to the constant overhead cost. Thirdly, owing to the simplicity of the optical networks used in this scheme, mode matching should be relatively straightforward. Fourthly, the scheme is inherently distributed: individual qubit-cavity systems can be placed in distant labs, and connected by optical fibers. This means that our scheme lends itself naturally to distributed applications, such as quantum repeaters 34 and quantum cryptography 35]. Finally, many of the techniques described here have been demonstrated experimentally, and the system requirements needed to create high-fidelity cluster states do not seem prohibitively restrictive.

While preparing this manuscript, we became aware of an alternative scheme that may also be used for generating cluster states of matter qubits [36]. We thank Tim Spiller and Bill Munro for valuable discussions and careful reading of the manuscript. The authors are supported by the E.U. Nanomagiq and Ramboq projects. head cost to the cluster generation process. For example, growing a 4-chain (without recycling) requires on average $p^{-3}+p^{-2}+p^{-1}$ applications of the EO, and each attempt to join such a chain adds on average $4 p-1$ qubits to the large cluster, leading to a total cost of $C_{4}=\left(p^{-3}+p^{-2}+p^{-1}+1\right) /(4 p-1)$ EOs per qubit added to the large cluster. A 5-chain can be grown by joining two 3-chains together. Joining to the end of a cluster, or by joining sub-chains together. Growing these $m$-chains adds a constant over-

FIG. 3: Cluster states with arbitrary single-qubit measure-
ments can perform a universal quantum computation. a) The qubits (circles) are entangled with their nearest horizontal neighbor via EOs (depicted by lines between the qubits), and gates between computational qubits are incorporated by the
vertical lines, b) Linear clusters can be joined together by applying the EO between qubits $A_{1}$ and $B_{1}$, and subsequently maining qubits are left in the state $\pm|C\rangle_{\{A\}}|\uparrow\rangle_{A_{1}}|C\rangle_{\{B\}} \pm$ $Z_{A_{2}}|C\rangle_{\{A\}}|\downarrow\rangle_{A_{1}} Z_{B_{2}}|C\rangle_{\{B\}}$, where the first sign depend ond sign depends on the outcome of the EO. Applying length $N+m-1$, of qubits $\left\{A_{N}, A_{1}, B_{2}, \ldots\right.$ the EO fails, qubit $A_{1}$ must be measured in the comby 1 qubit. Thus the average length of the new cluster is $=p(N+m-1)+(1-p)(N-1)$. In order that the clusthat length of the short chains should satisfy $m>1 / p$.

Chains of fixed length $m$ can be grown independently using the EO, either by sequentially adding single qubits

\section{.}
* Electronic address: sean.barrett@hp.com
$\dagger$ Electronic address: pieter.kok@hp.com
[1] J. Cirac and P. Zoller, Phys. Rev. Lett. 74, 4091 (1995).
[2] F. Jelezko et al., Phys. Rev. Lett. 92, 076401 (2004).
[3] E. Pazy et al., Europhys. Lett. 62, 175 (2003).
[4] A. Nazir et al., quant-ph/0403225 (2004).
[5] M. Riebe et al., Nature 429, 734 (2004). 
[6] M. D. Barrett et al., Nature 429, 737 (2004).

[7] C. Cabrillo et al., Phys. Rev. A 59, 1025 (1999).

[8] S. Bose et al., Phys. Rev. Lett. 83, 5158 (1999).

[9] X. L. Feng et al., Phys. Rev. Lett. 90, 217902 (2003).

[10] L.-M. Duan and H. J. Kimble, Phys. Rev. Lett. 90, 253601 (2003).

[11] D. Browne, M. Plenio, and S. Huelga, Phys. Rev. Lett. 91, 067901 (2003).

[12] C. Simon and W. T. M. Irvine, Phys. Rev. Lett. 91, 110405 (2003).

[13] I. E. Protsenko et al., Phy. Rev. A 66, 062306 (2002).

[14] X. Z. amd W. Mathis, quant-ph/0401042 (2004).

[15] L.-M. Duan et al., Quant. Inf. Comp. 4, 165 (2004).

[16] J. M. Taylor et al., cond-mat/0407640 (2004).

[17] R. Raussendorf and H. Briegel, Phys. Rev. Lett. 86, 5188 (2001).

[18] E. Knill, R. Laflamme, and G. Milburn, Nature 409, 26 (2001).

[19] N. Yoran and B. Reznik, Phys. Rev. Lett. 91, 037903 (2003).

[20] M. Nielsen, Phys. Rev. Lett. p. (to appear) (2004).

[21] D. Browne and T. Rudolph, quant-ph/0405157 (2004).

[22] H. Carmichael, An open systems approach to quantum optics, vol. 18 of Lecture notes in physics (Springer, Berlin, 1993).

[23] P. Kok and S. Braunstein, Phys. Rev. A 61, 042304 (2000).

[24] A. Beveratos et al., Eur. Phys. J. D 18, 191 (2002).

[25] T. A. Kennedy et al., Phys. Status Solidi (b) 233, 416 (2002).

[26] SPCM-AQR datasheet at www.perkinelmer.com.

[27] W. VonKlitzing et al., New J. Phys. 3, 14 (2001).

[28] Jason Smith, presonal communication.

[29] H.-A. Bachor and T. C. Ralph, An guide to experiments in quantum optics (Wiley-VCH, Weinheim, 2004).

[30] M. Pelton et al., Phys. Rev. Lett. 89, 233602 (2002).

[31] T. Stace, G. Milburn, and C. Barnes, Phys. Rev. B 67, 085317 (2003).

[32] F. Meier and D. D. Awschalom, cond-mat/0405342 (2004).

[33] J. McKeever et al., Science 303, 1992 (2004).

[34] H. Briegel et al., Phys. Rev. Lett. 81, 5932 (1998).

[35] A. Ekert, Phys. Rev. Lett. 67, 661 (1991).

[36] Y. Lim, A. Beige, and L. Kwek, to appear (2004). 